Resumen El presente artículo se propone analizar el origen y nacimiento del derecho humano a un medio ambiente sano y saludable con el objetivo de que todas las personas puedan vivir una vida digna y de calidad. Asimismo, analiza el contenido esencial desarrollo sostenible, que siempre ha de orientar al desarrollo del derecho medioambiental para poder garantizar un medio ambiente saludable a las generaciones humanas presentes y futuras, y un crecimiento económico sostenible que contribuya a desarrollar la igualdad de oportunidades de todas las personas.

Abstract

This article analyses the origin and birth of the human right to a safe and healthy environment in order to allow everyone to live a dignified and quality life. It also analyses the essential content of sustainable development, which must always guide the development of environmental law to ensure a healthy environment for human present and future generations, and a sustainable economic growth that contributes to the development of equal opportunities for all people.

Key words Derecho medioambiental; desarrollo sostenible; medio ambiente saludable; igualdad de oportunidades.

Environmental law; sustainable development; healthy environment; equal opportunities. 


\section{Introducción}

El derecho humano medioambiental busca garantizar una calidad de vida a todas las personas en un medio ambiente sano y saludable, tanto de las generaciones presentes como de las generaciones futuras. En consecuencia, la aplicabilidad y exigibilidad del derecho medioambiental por parte de todas las personas ha de ser una constante en todas las políticas sociales y económicas llevadas a cabo, pues los seres humanos para poder vivir una vida digna y de calidad precisan de un entorno o medio ambiente sano y saludable, racionalizando el uso de los recursos naturales y humanos.

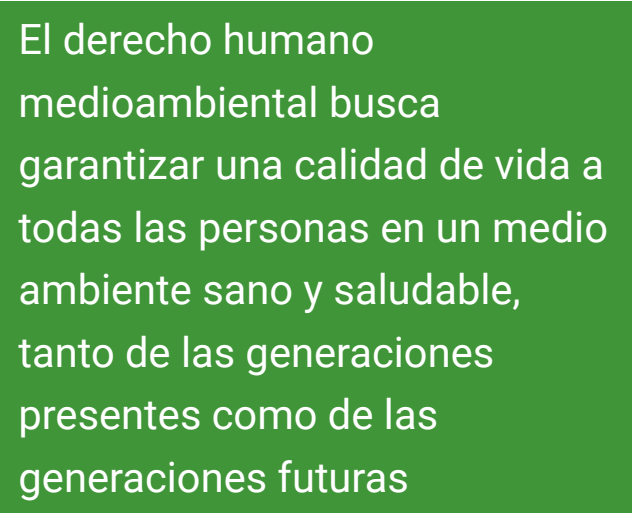

El objetivo principal del presente artículo es analizar el nacimiento y el desarrollo posterior del derecho humano medioambiental en las últimas décadas del pasado siglo, enmarcado dentro de la cuarta generación de los derechos humanos del tercer milenio, que ha de ir unido inexorablemente al concepto de desarrollo sostenible.

El desarrollo sostenible consiste en un proceso de desarrollo socioeconómico capaz de prolongarse en el tiempo sin minar catastróficamente la capacidad de la naturaleza para mantenerlo, a través de un consumo eficiente y razonable de los recursos naturales limitados. Por consiguiente, el desarrollo sostenible ha de poder garantizar la satisfacción de las necesidades básicas de todos los seres humanos, en cualquier lugar del mundo, asegurando a su vez la igualdad de oportunidades de todas las personas, el aumento del potencial productivo del crecimiento socioeconómico, y contribuir de esta manera a la erradicación de la pobreza en el mundo.

Por último, este artículo pretende analizar la necesidad de los límites entre la economía y el medio ambiente, que se encuentran íntimamente interrelacionados, con el objetivo esencial de establecer límites entre ellos en un periodo de tiempo determinado, para que no se produzca la expansión indiscriminada del desarrollo económico a costa del deterioro del medio ambiente, sino que el desarrollo sostenible sea el faro que señale los límites al crecimiento económico sin deteriorar el medio ambiente de las generaciones presentes y futuras.

\section{El derecho medioambiental}

En la actualidad, el "derecho medioambiental" o derecho al medio ambiente de las personas es considerado como un derecho humano que pertenece a la "cuarta generación" de los derechos humanos del tercer milenio y se podría definir como: el derecho a disfrutar de un medio ambiente sano, enmarcado dentro del derecho a la calidad de vida de todas las personas.

Los derechos humanos de cuarta generación fueron naciendo a partir de la expansión y consolidación del Estado del bienestar en los países desarrollados, durante las últi- 
mas décadas de la segunda mitad del siglo XX, íntimamente unidos al impulso de las reivindicaciones de los distintos grupos de presión de ciudadanos que buscaban poder disfrutar de una mejor calidad de vida medioambiental. En consecuencia, estos nuevos derechos humanos son producto de un cambio social que ya se estaba fraguando en los nuevos Estados sociales, democráticos y de derecho en los países desarrollados durante la segunda mitad del siglo pasado, y que se pueden con-

El derecho medioambiental se

encontraría enmarcado dentro

del grupo de los derechos

relativos a la protección del

ecosistema y al patrimonio de la

humanidad siderar a todas luces como los nuevos derechos humanos del tercer milenio.

El derecho medioambiental se encontraría enmarcado dentro del grupo de los derechos relativos a la protección del ecosistema y al patrimonio de la humanidad. Este derecho humano estaría compuesto por varios derechos que complementarían su verdadera esencia y desarrollo, y cuyo último objetivo sería garantizar un medio ambiente compatible con la vida humana y el desarrollo económico y social, protegiendo la calidad de vida de las personas.

En cuanto al derecho al medio ambiente como derecho humano, Gómez Sánchez (2004, pp. 243-244) considera que el derecho medioambiental integra no solo el derecho medioambiental en sí mismo considerado, sino que aparece conformado por otros derechos relativos al patrimonio cultural de la humanidad, como serían entre otros:

- El derecho al mantenimiento y desarrollado equilibrado del hábitat.

- El derecho a la obtención de alimentos que no alteren ni pongan en peligro la identidad genética ni la salud humana.

- El derecho de acceso a los entornos naturales.

- El derecho al reconocimiento y protección del patrimonio cultural.

- El derecho a un desarrollo industrial y tecnológico que, sin perjuicio del progreso de la sociedad, resulte compatible con el más prioritario progreso de la especie humana.

- El derecho a la obtención de productos industriales y farmacéuticos que no alteren ni menoscaben la integridad ni la identidad del ser humano.

En la actualidad, uno de los problemas más inquietante y grave para la humanidad es el deterioro medioambiental que agudiza el peligro de la supervivencia de la propia especie humana. Pérez Luño (2006, p. 30) considera que "la humanidad puede estar abocada al suicidio colectivo porque con un progreso técnico irresponsable ha desencadenado las fuerzas de la naturaleza y no se halla en condiciones de controlarlas". Por lo tanto, el problema es de tal gravedad, magnitud e importancia, que las instituciones políticas, sociales y económicas así como la sociedad en su conjunto, han de navegar 
al unísono en la misma dirección para detener y erradicar el deterioro del medio ambiente, que se erige en nuestro propio hábitat y el de las generaciones futuras.

En el Pacto de los Derechos Económicos, Sociales y Culturales de 1966, en su artículo 12.2.b, se hace alusión a la necesidad del mejoramiento del medio ambiente. No obstante, es la Declaración de Estocolmo de 16 de junio de 1972 (Declaración de la Conferencia de las Naciones Unidas sobre Medio Ambiente y Desarrollo Humano) la primera que proclama el derecho humano al medio ambiente al establecer que:

El hombre tiene el derecho fundamental a la libertad, a la igualdad y al disfrute de condiciones de vida adecuadas en un medio de calidad que le permita llevar una vida digna y gozar del bienestar y tiene la solemne obligación de proteger y mejorar el medio para las generaciones presentes y futuras (Conferencia de las Naciones Unidas sobre Medio Ambiente y Desarrollo Humano, 1972, p. 4).

Por consiguiente, el derecho a

un medio ambiente sano ha de

conformarse como un auténtico

derecho humano dentro

del grupo de los derechos

ecológicos
En las últimas décadas del pasado siglo, el derecho medioambiental se fue consolidando y desarrollando en diversos protocolos y conferencias internacionales, como fueron entre las más destacables: el Protocolo de Montreal, de 16 de septiembre de 1987, relativo a las Sustancias que agotan la capa de ozono; el artículo 24 de la Carta Africana de los Derechos del Hombre y de los Pueblos, de 12 de agosto de 1992; y sobre todo, la Declaración sobre Medio Ambiente y Desarrollo, elaborada en la II Conferencia Mundial de las Naciones Unidas, celebrada en Río de Janeiro entre el 3 y el 14 de junio de 1992, cuyo tercer principio afirma que el derecho al desarrollo ha de ejercerse en forma tal que responda equitativamente a las necesidades de desarrollo y necesidades ambientales de las generaciones presentes y futuras. No obstante, esta última declaración no preveía sanciones aplicables a los países que incumplieran las medidas adoptadas en la Conferencia de Río de Janeiro, por lo que el 11 de diciembre de 1997 se elaboró el Convenio Marco de las Naciones Unidas sobre el Cambio Climático, conocido comúnmente por el Protocolo de Kioto, que no fue ratificado por todos los Estados miembros de las Naciones Unidas, como fue el caso de Estados Unidos, por lo que las sanciones no se pudieron aplicar de una forma efectiva a todos los países, siendo sus resultados escasos o incluso nulos en algunas situaciones.

La exigibilidad del derecho medioambiental por parte de todas las personas ha de ser una constante en todas las políticas sociales y económicas llevadas a cabo, pues los seres humanos para poder vivir una vida digna y de calidad precisan de un entorno o medio ambiente sano y saludable, racionalizando el uso de los recursos naturales y humanos. Por consiguiente, el derecho a un medio ambiente sano ha de conformarse como un auténtico derecho humano dentro del grupo de los derechos ecológicos.

No podemos olvidar la íntima conexión que ha de existir entre el derecho humano a un medio ambiente sano y el desarrollo sostenible, basado en la garantía del derecho 
medioambiental de las generaciones futuras. Ahondando en este análisis garantizador del derecho medioambiental y su problemática social, Martínez Morán enfatiza que:

\begin{abstract}
Uno de los aspectos importantes que hay que tener en cuenta en el debate general sobre el derecho al medio ambiente es su vinculación o relación con el desarrollo sostenible y con los derechos de las generaciones futuras. En efecto el derecho a un medio ambiente sano tiene mucho más calado del que a primera vista parece pues es cierto que las generaciones presentes de seres humanos tenemos derecho a una calidad de vida y a la satisfacción de un cúmulo de necesidades para lo cual nos servimos de cuanto nos aporta la naturaleza a la que de algún modo tenemos que explotar. Pero no es menos cierto que, al mismo tiempo, que debe satisfacerse el derecho a la calidad de vida y al progreso (...) debemos tener en cuenta que dicha satisfacción es incompatible con el saqueo y sobreexplotación de la naturaleza en aras de respetar y posibilitar la satisfacción de las necesidades y derechos de las generaciones futuras (Martínez Morán, 2014, p. 128).
\end{abstract}

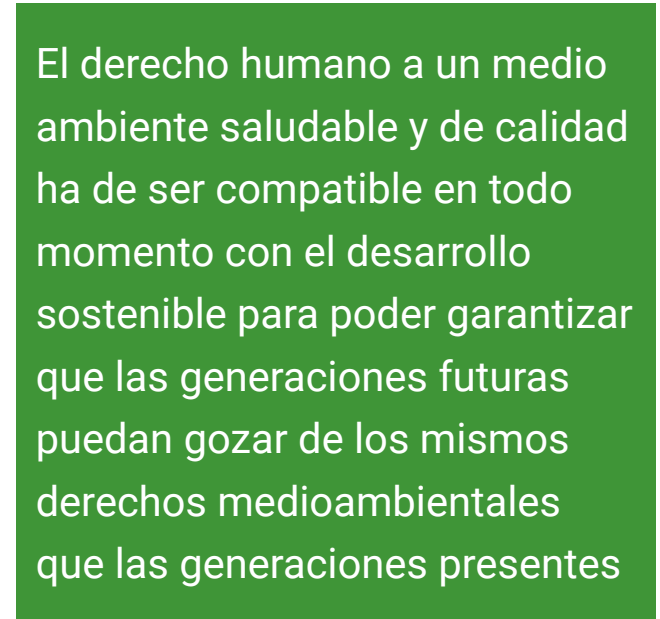

En conclusión, el derecho humano a un medio ambiente saludable y de calidad ha de ser compatible en todo momento con el desarrollo sostenible para poder garantizar que las generaciones futuras puedan gozar de los mismos derechos medioambientales que las generaciones presentes, pues no podemos hipotecar la calidad del medio ambiente de nuestros hijos y nietos que han de ser nuestros herederos, para que puedan gozar de un medio ambiente sano y una vida de calidad en el mismo planeta Tierra que nosotros habitamos en estos momentos.

\title{
3. El desarrollo sostenible
}

El término de "desarrollo sostenible" se acuñó por vez primera en la cumbre de la Tierra de Río de Janeiro de 1992, conocido comúnmente como "desarrollo sostenible o sustentable", derivado de la palabra inglesa sustainable, que viene a definirse como: "El proceso de desarrollo socioeconómico capaz de prolongarse en el tiempo sin minar catastróficamente la capacidad de la naturaleza para mantenerlo" (García García, 2004, p. 145).

En 1983, las Naciones Unidas crearon la Comisión Mundial sobre el Medio Ambiente y el Desarrollo, que fue presidida por Brundtland. En 1987, se publicó el informe de la comisión (CMMAD 1988) en el que se acuñó el término de "desarrollo sostenible", impulsándolo definitivamente en el ámbito internacional, la Conferencia de Río de Janeiro, en 1992.

El principio de la solidaridad intergeneracional del desarrollo económico y humano, así como su visión futura a largo plazo, fue remarcado por la propia Comisión Mundial sobre el Medio Ambiente y el Desarrollo, definiéndolos con las siguientes palabras: "Está en manos de la humanidad hacer que el desarrollo sea sostenible, es decir, asegurar que satisfaga las necesidades del presente sin comprometer la capacidad de las futuras generaciones para satisfacer las propias" (CMMAD, 1988, p. 29). No obstante, es necesario destacar que tanto en la realidad histórica como en el momento actual es 
muy difícil poder actuar o dejar de actuar en el presente sin llegar a comprometer de alguna manera a las generaciones futuras.

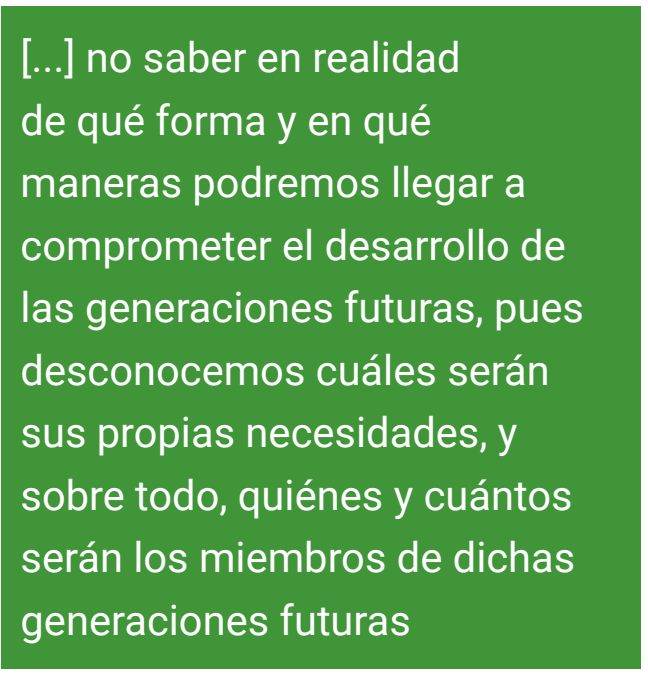

La polémica y complejidad del concepto y contenido del desarrollo sostenible consiste en parte en no saber en realidad de qué forma y en qué maneras podremos llegar a comprometer el desarrollo de las generaciones futuras, pues desconocemos cuáles serán sus propias necesidades, y sobre todo, quiénes y cuántos serán los miembros de dichas generaciones futuras. Además, existe una amplia ambigüedad política en el concepto, pues como sostiene García: "al exigir que el desarrollo llegue a ser sustentable, reconoce implícitamente que ahora no lo es, sugiriendo que algo va mal en el modelo social vigente y que resulta conveniente reformarlo" (García García, 2004, p. 147).

Las ideologías políticas de izquierdas y de derechas se enfrentan al término desde diferentes ópticas; mientras que las primeras consideran que el desarrollo actual ha de ser más social o humano, luchando por su reforma o corrección; las segundas consideran que no existe ninguna alternativa factible al modelo de desarrollo actual vigente. Por consiguiente, según afirma García:
La izquierda y la derecha pueden reconocerse en la palabra mágica, los verdes pue-
den verla como prueba de la legitimidad social de sus denuncias y los productivistas
como confirmación de que, en lo esencial, sus prioridades no requieren más que
algunos ajustes. La buena nueva actúa en este doble frente: "desarrollo" es la reafir-
mación, el recordatorio de que el camino seguido ha sido acertado; "sostenible" es la
promesa de un futuro sin restricciones ni decadencias (García García, 2004, p. 147).

El término de desarrollo sostenible es un término en el que se aprecia una gran vaguedad e imprecisión en cuanto a su definición. Esta imprecisión le permite adaptarse a ideologías diferentes, tanto de derechas como de izquierdas, es decir, establecer líneas de conexión entre los partidarios del desarrollo actual imperante y los ecologistas, con el objetivo último de perdurar en el tiempo el crecimiento económico vigente y el desarrollo humano en el mundo globalizado.

El desarrollo sostenible ha de poder garantizar la satisfacción de las necesidades básicas de todos los seres humanos, en cualquier lugar del mundo, asegurando a su vez la igualdad de oportunidades de todas las personas, el aumento del potencial productivo del crecimiento socioeconómico, y contribuir a la erradicación de la pobreza en el mundo. En consecuencia, según establece la CMMAD, el concepto de desarrollo sostenible comprendería varias acepciones y objetivos:

La satisfacción de las necesidades y aspiraciones humanas es el principal objetivo del desarrollo. En los países en desarrollo no se satisfacen las necesidades esen- 
ciales -alimento, ropa, abrigo, trabajo- de gran número de personas, que tienen además legítimas aspiraciones a una mejor calidad de vida. Un mundo en el que la pobreza y la desigualdad son endémicas será siempre propenso a sufrir crisis ecológicas o de otra índole. El desarrollo sostenible requiere la satisfacción de las necesidades básicas de todos y extiende a todos la oportunidad de satisfacer sus aspiraciones a una vida mejor (CMMAD, 1988, pp. 68-70).

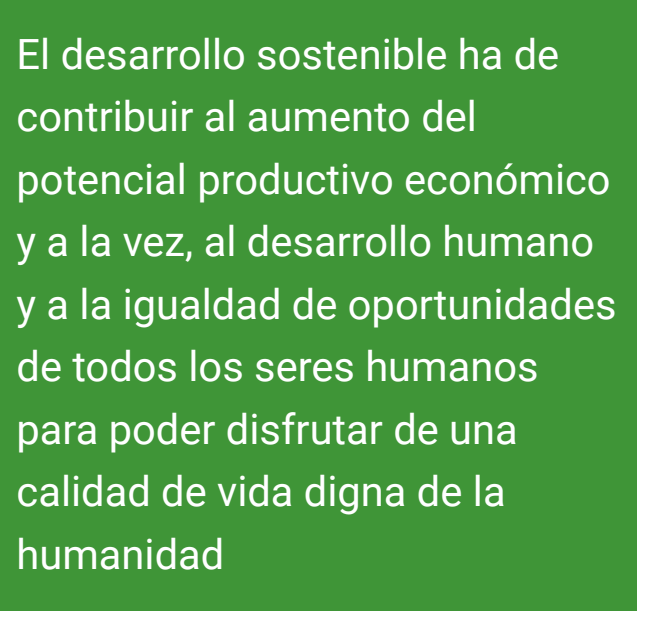

Los niveles de vida de todas las personas serán sostenibles si pensamos y aplicamos el desarrollo socioeconómico a largo plazo a través de un consumo responsable y razonable. Debemos desarrollar el crecimiento económico de las zonas geográficas del mundo en las que no se cubren ni satisfacen adecuadamente las necesidades básicas de sus poblaciones. Por consiguiente, el desarrollo sostenible ha de contribuir al aumento del potencial productivo económico y a la vez, al desarrollo humano y a la igualdad de oportunidades de todos los seres humanos para poder disfrutar de una calidad de vida digna de la humanidad.

La explotación de los recursos naturales ha de realizarse de acuerdo con el cambio del paradigma social, político y económico, y según el desarrollo sostenible, pues como sostiene la CMMAD:

\begin{abstract}
El desarrollo sostenible es un proceso de cambio en el cual la explotación, la orientación de la evolución tecnológica y la modificación de las instituciones están acordes y acrecientan el potencial actual y futuro para satisfacer las necesidades y aspiraciones humanas (CMMAD, 1988, pp. 68-70).
\end{abstract}

Asimismo, el desarrollo sostenible no implica la paralización del crecimiento económico globalizado, pues no sería posible erradicar la pobreza en el mundo, ni el subdesarroIlo, ni los problemas medioambientales sin un eficaz crecimiento económico, tal y como afirmó la ONU en su informe sobre el desarrollo económico, social y medioambiental:

\footnotetext{
El desarrollo sustentable no implica el cese del crecimiento económico. Más bien exige el reconocimiento de que los problemas de la pobreza y el subdesarrollo, y los problemas ambientales relacionados, no se pueden resolver sin un vigoroso crecimiento económico. No obstante, el desarrollo sustentable requerirá cambios en las formas actuales de crecimiento, para hacerlas menos intensivas en energía y recursos, y más equitativas (ONU, 1990, p. 75).
}

Por lo tanto, según las Naciones Unidas, el desarrollo sostenible precisa de su integración dentro del entramado económico y productivo actual, con el objetivo esencial de no limitar el crecimiento económico mundial sino de que este sea más eficiente y 
sostenible tanto en el corto como en el largo plazo, desarrollando cambios sustanciales en el crecimiento económico en su relación estrecha e íntima con el medio ambiente.

Las diversas acepciones teóricas y de análisis del desarrollo sostenible son muy numerosas, abarcando diferentes perspectivas como serían: la económica, social o cultural, entre otras. García sostiene que las distintas opciones o alternativas podrían reducirse principalmente a tres, y serían las siguientes:

a. El desarrollo sostenible entendido como un crecimiento sostenido, manteniendo la expansión de la producción y el consumo, consolidando una cultura de acumulación de bienes materiales, supeditando la reducción de la desigualdad a la creación de más riqueza a repartir y reforzando la dependencia a escala mundial. La innovación tecnológica habría de asegurar la inocuidad de los eventuales episodios de escasez o deterioro de los recursos materiales.

b. El desarrollo sostenible entendido como mejora cualitativa sin incremento de la escala física, es decir, como evolución de una economía homeostática, en estado estacionario o de crecimiento cero.

c. La sostenibilidad, siempre incierta y sujeta a la necesidad de una permanente adaptación a condiciones azarosas, solamente podría conseguirse a condición de abandonar el desarrollo, causa tanto de la pobreza como de la degradación del medio ambiente (García García, 2004, pp. 154-155).

Asimismo, sería necesario

destacar la variabilidad en

el tiempo de los gustos o

preferencias y del consumo

de las personas, tanto a nivel

individual como colectivo,

pues son factores íntimamente

relacionados con el tiempo, su

evolución y por tanto, con la

sostenibilidad del desarrollo
Las anteriores alternativas no son excluyentes entre sí, sino que se encuentran íntimamente relacionadas entre ellas, considerando que el concepto de sostenibilidad hace alusión a la relación directa y estrecha entre las poblaciones y las fuentes de energía y recursos naturales del ecosistema en el que se desarrollan. Por consiguiente, sería necesario considerar y relacionar el desarrollo sostenible de una población y el medio ambiente en el que se desenvuelve, pues según afirma García: "Un proceso de desarrollo social es ecológicamente sostenible (durante un periodo de tiempo determinado) si puede mantenerse dentro de la capacidad de carga de su ecosistema o medio ambiente, pero eso siempre para una determinada media de nivel de vida" (García, 2004, p. 159).

Otro de los factores claves del desarrollo sostenible que deberíamos considerar sería el factor tiempo, pues se torna imprescindible en el análisis de la sostenibilidad del desarrollo, basado en el principio de que nada puede crecer ni durar eternamente en un medio finito (García, 2004, p. 159). Los cambios tecnológicos que se suceden en el tiempo en las sociedades, aceleran la eficiencia en el empleo de los recursos materiales y naturales, aumentando su utilidad real o potencial para las personas. Asimismo, sería necesario destacar la variabilidad en el tiempo de los gustos o preferencias y del consumo de las personas, tanto a nivel individual como colectivo, pues son factores íntimamente relacionados con el tiempo, su evolución y por tanto, con la sostenibilidad del desarrollo. 
Por último, hemos de considerar que los recursos naturales son limitados, por lo tanto su explotación ha de encaminarse necesariamente de la mano del desarrollo sostenible. El crecimiento actual de las economías de los países del mundo globalizado, depende estrechamente de la necesidad de no agotar los recursos naturales ni saturar los vertederos de residuos. En consecuencia, la economía y el medio ambiente, que se encuentran íntimamente interrelacionados han de establecer límites entre ellos para que no se produzca la expansión indiscriminada del desarrollo económico a costa del deterioro del medio ambiente, sino que el desarrollo sostenible sea el faro que señale los límites al crecimiento económico sin deteriorar el medio ambiente de las generaciones presentes y futuras.

\section{Conclusiones}

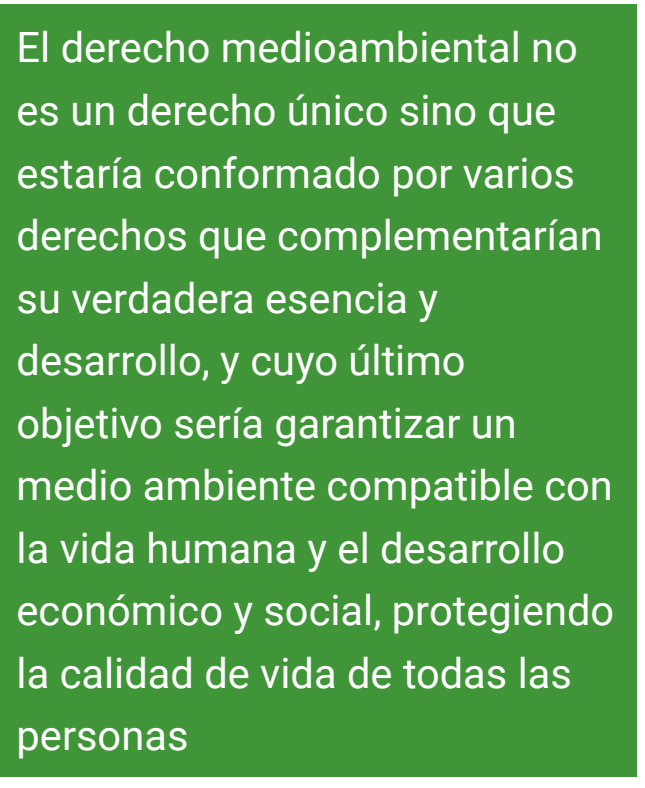

En el presente artículo hemos podido analizar el nacimiento, en las últimas décadas de la segunda mitad del pasado siglo, del derecho humano a un medio ambiente sano y saludable con el objetivo de que todas las personas puedan vivir una vida digna y de calidad. A su vez, hemos analizado el origen y el contenido esencial del desarrollo sostenible, que siempre ha de orientar al derecho medioambiental para poder garantizar un medio ambiente sano y saludable no solo a las generaciones presentes sino también a las generaciones futuras.

En el análisis del presente artículo hemos de establecer las siguientes conclusiones:

La primera nos lleva a considerar al derecho humano medioambiental como un derecho humano de todas las personas, perteneciente a la cuarta generación de los derechos humanos del tercer milenio, y se encuentra enmarcado dentro del grupo de los derechos humanos relativos a la protección del ecosistema y al patrimonio de la humanidad.

La segunda constata que el derecho medioambiental no es un derecho único sino que estaría conformado por varios derechos que complementarían su verdadera esencia y desarrollo, y cuyo último objetivo sería garantizar un medio ambiente compatible con la vida humana y el desarrollo económico y social, protegiendo la calidad de vida de todas las personas.

La tercera se centraría en el propio concepto del desarrollo sostenible en cuyo término se aprecia una gran vaguedad e imprecisión. No obstante, es esta imprecisión y complejidad del término lo que le permite adaptarse a las ideologías diferentes y poder establecer líneas de conexión entre los partidarios del desarrollo actual imperante y los ecologistas, con el objetivo último de perdurar en el tiempo, inmerso en el crecimiento económico vigente y el desarrollo humano en un mundo globalizado.

La última conclusión se enmarcaría en el camino hacia la transformación más o menos acentuada del modelo socioeconómico imperante, con la esperanza de lograr un 
desarrollo económico y humano más eficiente y racional, compatible con el respeto al medio ambiente. Respeto que ha de darse tanto a corto como a largo plazo, a través de un consumo responsable y razonable, desarrollando el crecimiento económico de las zonas geográficas del mundo en las que no se cubren ni satisfacen adecuadamente las necesidades básicas de sus poblaciones. Debemos impulsar de esta forma el desarrollo humano y la igualdad de oportunidades de todas las personas, para poder disfrutar y garantizar una calidad de vida digna tanto a las generaciones presentes como a las generaciones venideras, herederas de nuestro sistema económico vigente y del estado actual del medio ambiente.

\section{Bibliografía}

CMMAD (Comisión Mundial del Medio Ambiente y del Desarrollo) (1988). Nuestro futuro común. Madrid: Alianza.

Conferencia de las Naciones Unidas sobre Medio Ambiente y Desarrollo Humano (1972). Estocolmo, 3 al 14 de junio de 1972 (A/CONF.48/14/REV.1).

Conferencia de las Naciones Unidas sobre el Medio Ambiente y Desarrollo (1993). Río 92. Programa 21. Tomo II. Madrid, Ministerio de Obras Públicas y Transportes, Dirección General de Política Ambiental.

García García, J. E. (2004). Medio ambiente y sociedad. La civilización industrial y los límites del planeta. Madrid: Alianza ensayo.

Gómez Sánchez, Y. (2004). Estado Constitucional y protección internacional. Comisión Nacional de los Derechos Humanos. En Gómez Sánchez, Y. (coord.). Pasado, presente y futuro de los derechos Humanos. México: CNDDHH-UNED.

Martínez Morán, N. (2014). Capítulo VI. La Tercera generación de derechos Humanos: Derechos colectivos: la solidaridad. En Marcos del Cano, A. M. (coord.). Derechos Humanos y Trabajo Social. Madrid: Universitas.

Pérez Luño, A. E. (2006). La tercera generación de Derechos Humanos. Navarra: Thomsom-Aranzadi.

ONU (1990). Global outlook 2000: Economic, social, environmental. Nueva York: United Nations Publications. 